\title{
Associations Between Rural or Urban Status, Health Outcomes and Behaviors, and COVID-19 Perceptions Among Meditation App Users: Longitudinal Survey Study
}

Nishat Bhuiyan ${ }^{1}$, PhD; Megan Puzia ${ }^{2}$, MS; Chad Stecher ${ }^{1}$, PhD; Jennifer Huberty ${ }^{1}, \mathrm{PhD}$

${ }^{1}$ College of Health Solutions, Arizona State University, Phoenix, AZ, United States

${ }^{2}$ Behavioral Research and Analytics, LLC, Salt Lake City, UT, United States

Corresponding Author:

Nishat Bhuiyan, $\mathrm{PhD}$

College of Health Solutions

Arizona State University

500 North 3rd Street

Phoenix, AZ, 85004

United States

Phone: 14804062644

Email: nbhuiyan@asu.edu

\section{Abstract}

Background: Rural and urban differences in health outcomes and behaviors have been well-documented, with significant rural health disparities frequently highlighted. Mobile health (mHealth) apps, such as meditation apps, are a novel method for improving health and behaviors. These apps may be a critical health promotion strategy during the COVID-19 pandemic and could potentially be used to address rural health disparities. However, limited research has assessed whether meditation app health outcomes are associated with rural and urban residence, and it is unclear whether disparities in health and behaviors between rural and urban populations would persist among meditation app users.

Objective: We aimed to explore associations between rural or urban status, psychological outcomes, and physical activity among users of a mobile meditation app. We further aimed to explore associations between rural or urban status and perceived effects of COVID-19 on stress, mental health, and physical activity, and to explore changes in these outcomes in rural versus urban app users over time.

Methods: This study was a secondary analysis of a national survey conducted among subscribers to the meditation app Calm. Eligible participants completed online baseline surveys from April to June 2020, and follow-up surveys from June to September 2020, assessing demographics, psychological outcomes, physical activity, and perceived effects of COVID-19 on stress, mental health, and physical activity.

Results: Participants ( $=8392)$ were mostly female (7041/8392, 83.9\%), non-Hispanic (7855/8392, 93.6\%), and White (7704/8392, 91.8\%); had high socioeconomic status (income $\geq$ US \$100,000: 4389/8392, 52.3\%; bachelor's degree or higher: 7251/8392, 86.4\%); and resided in a metropolitan area core (rural-urban commuting area code 1: 7192/8392, 85.7\%). Rural or urban status was not associated with baseline stress, depression, anxiety, pre-COVID-19 and current physical activity, or perceived effects of COVID-19 on stress, mental health, and physical activity. Repeated-measures models showed overall decreases in depression, anxiety, and perceived effects of COVID-19 on physical activity from baseline to follow-up, and no significant changes in stress or perceived effects of COVID-19 on stress and mental health over time. Models also showed no significant main effects of rural or urban status, COVID-19 statewide prevalence at baseline, or change in COVID-19 statewide prevalence.

Conclusions: We did not find associations between rural or urban status and psychological outcomes (ie, stress, depression, and anxiety), physical activity, or perceived effects of COVID-19 on stress, mental health, and physical activity. Rural or urban status does not appear to drive differences in outcomes among meditation app users, and the use of mHealth apps should continue to be explored as a health promotion strategy in both rural and urban populations. Furthermore, our results did not show negative cumulative effects of COVID-19 on psychological outcomes and physical activity among app users in our sample, the majority of whom were urban, White, female, and of high socioeconomic status. Further research is needed to investigate meditation app use as a health promotion strategy in rural and urban populations. 
(JMIR Mhealth Uhealth 2021;9(5):e26037) doi: 10.2196/26037

\section{KEYWORDS}

mHealth; rural health; physical activity; mental health; COVID-19

\section{Introduction}

Significant rural and urban status-related differences in health outcomes and behaviors have been well-documented in the United States [1-6]. Some studies have demonstrated poorer outcomes in urban areas, such as increased risk for certain mental disorders and cancer incidence [1,2]. However, for the majority of health outcomes and behaviors, rural residents face poorer outcomes compared to their urban counterparts, including higher chronic disease incidence and mortality rates and lower diet quality and physical activity [3-6], underscoring the need for methods to address rural health disparities.

The use of mobile health (mHealth) apps has been rapidly growing as a novel method for health promotion [7,8], as strategies that include mHealth apps may be more cost-effective and scalable as well as have wider reach than in-person-delivered programs [9]. mHealth tools may be even more critical during the current COVID-19 pandemic, given the limited availability and potential risks associated with in-person-delivered programs [10]. Studies have demonstrated that mHealth apps can effectively improve mental and physical health and behaviors [7,8]. Rural areas in the United States are often characterized by limitations in resources, such as medical facilities and specialty health care services; thus, the use of mHealth apps to remotely deliver health interventions to improve outcomes in rural populations may be of paramount importance to rural communities and help to address ongoing rural health disparities [11,12].

The use of meditation apps, in particular, could also be used to potentially address disparities in mental health outcomes and health behaviors faced by rural residents. Meditation is a well-known strategy for improving mental health outcomes, such as stress, depression, and anxiety [13,14]. Furthermore, growing literature further suggests that meditation is a promising tool for counteracting sedentariness to address physical inactivity [15]. Given the poorer mental health outcomes [16,17], lower access to mental health treatment [17], and lower prevalence of positive health outcomes and behaviors among rural residents [18], research exploring rural and urban status-related differences in health and behavior outcomes among meditation app users is warranted.

Limited research has assessed whether mHealth app outcomes are associated with rural and urban residence, and it is currently unclear whether disparities in health and behaviors between rural and urban populations would persist among mobile meditation app users. Thus, the purpose of this study is to (1) explore associations between rural or urban status, psychological outcomes, and physical activity; (2) explore associations between rural or urban status and perceived effects of COVID-19 on stress, mental health, and physical activity; and (3) assess changes in psychological outcomes, physical activity, and perceived effects of COVID-19 over time among rural versus urban users of a mobile meditation app.

\section{Methods}

\section{Overview}

This study was a secondary analysis of a national survey conducted in a nonrandom convenience sample of paying subscribers to the mobile meditation app Calm. All study materials and procedures were reviewed and approved by the Institutional Review Board at Arizona State University (protocol ID: STUDY00014534). Potentially eligible subscribers met the following inclusion criteria: (1) were 18 years of age or older, (2) had opened an email from Calm and used Calm at least once in the last 90 days, (3) were able to read and understand English, and (4) were United States residents. Potentially eligible subscribers were identified by the Calm informatics team and were sent a study recruitment email by the research team. The recruitment email included a brief study description and the link to an online Qualtrics eligibility survey to verify that they met the following inclusion criteria: (1) were at least 18 years of age, (2) were able to read and understand English, and (3) resided in the United States or a United States territory. This survey was free and voluntary and took approximately 3 minutes to complete.

Eligible participants were emailed a link to complete an electronic informed consent form, which stated the study purpose; the identity of the investigator; the length of time to complete the survey; which data were stored, where, and for how long; potential risks and benefits; and compensation details. Eligible participants were emailed links to online Qualtrics baseline and follow-up surveys. Surveys took approximately 15 minutes to complete and were free and voluntary; in addition, participants were able to skip survey questions.

The time frame for this study was approximately 2 months, from baseline to follow-up. Baseline surveys were distributed from April 22 to June 3, 2020, and follow-up surveys were distributed from June 26 to September 11, 2020. To maintain participant confidentiality, study data were imported into a secure and backed-up MySQL (Structured Query Language) database. Each database user had their own username and password, with permissions set appropriately for that user. Query logging was enabled to provide an electronic audit trail that recorded all user interactions with the database. The database did not store personally identifiable information; instead, records were linked to individual participants via a unique participant ID. Regarding compensation, all participants were entered into a random drawing for 1 of 20 gift cards valued at US $\$ 50.00$ for completing the baseline survey (ie, 20 participants received US \$50.00 at baseline) and a random drawing for 1 of $20 \mathrm{gift}$ cards valued at US $\$ 50.00$ for completing the follow-up survey (ie, 20 participants received US $\$ 50.00$ at follow-up). 


\section{Measures}

The online Qualtrics baseline and follow-up surveys included both investigator-developed and validated questionnaires. Participants self-reported demographic characteristics; stress, via the Perceived Stress Scale [19]; depression and anxiety, via the Hospital Anxiety and Depression Scale (HADS) [20]; pre-COVID-19 and current physical activity (days/week); and the extent to which COVID-19 affected their stress, mental health, and physical activity. The Perceived Stress Scale includes 10 items that measure the degree of self-appraised stress in one's life within the past month [19]. Response items are scored on a 5-point Likert scale ranging from 0 (never) to 4 (very often). Items are summed to produce a total score ranging from 0 to 40 , with higher scores indicating higher levels of perceived stress. The Perceived Stress Scale is a reliable and valid measure that has demonstrated good internal consistency (Cronbach $\alpha=$.74-.91) [19]. The HADS is a 14-item scale measuring levels of anxiety and depression. Seven items comprise the anxiety subscale (HADS-A) and seven items comprise the depression subscale (HADS-D) [20]. Response items are scored on a 4-point Likert scale ranging from 0 to 3 . Items are summed to produce a total score ranging from 0 to 21 for each subscale. Scores between 0 and 7 are considered normal, scores from 8 to 10 are considered borderline abnormal, and scores from 11 to 21 are considered abnormal. The HADS is a valid and reliable tool, with internal consistency reported to reach $\alpha$ levels of .93 and .90 for the HADS-A and HADS-D subscales, respectively [20]. Participants were asked to self-report how many days per week (on a scale of 0 to 7 ) of physical activity they participated in prior to COVID-19 as well as their current participation. Participants were asked, via investigator-developed items, "To what extent do you feel the COVID-19 pandemic has affected your stress?" "To what extent do you feel the COVID-19 pandemic has affected your mental health?" and "To what extent do you feel the COVID-19 pandemic has affected your physical activity?" Response items were scored on a 5-point Likert scale, ranging from 0 (not at all) to 4 (very much), with higher summed scores representing a greater impact of COVID-19 on outcomes.

Participant ZIP Codes were categorized into levels of urbanization using the US Department of Agriculture's rural-urban commuting area (RUCA) codes, which range from 1 , indicating a metropolitan core area, to 10 , indicating a rural area [21]. RUCA code definitions can be found in Table 1. To define rural or urban status, a binary variable was created by dummy-coding RUCA 1 as urban and RUCA 2 to 10 as rural. As an alternative approach, we also treated the RUCA code as a continuous variable in replicated study analyses, the results of which can be found in Multimedia Appendix 1.

COVID-19 prevalence data were derived from the US Centers for Disease Control and Prevention aggregate data set of daily numbers of confirmed and probable case and deaths over time [22]. This information was used to categorize each state's relative COVID-19 risk, where low-prevalence COVID-19 states were defined as those with fewer than 10,000 cases per 100,000 at the time of survey distribution and high-prevalence COVID-19 states were those with 10,000 cases or more per 100,000. At baseline, high-prevalence COVID-19 states included California, Colorado, Connecticut, Florida, Georgia, Illinois, Indiana, Louisiana, Massachusetts, Maryland, Michigan, New Jersey, New York, Ohio, Pennsylvania, Texas, Virginia, and Washington. At follow-up, high-prevalence COVID-19 states additionally included Alabama, Arkansas, Arizona, DC, Delaware, Iowa, Kansas, Kentucky, Minnesota, Missouri, Mississippi, North Carolina, Nebraska, New Mexico, Nevada, Oklahoma, Rhode Island, South Carolina, Tennessee, Utah, and Wisconsin. A binary variable, COVID-19 state baseline, was created based on high-prevalence COVID-19 states, and a second variable, COVID-19 state change, was created based on states that were low prevalence at baseline and changed to high prevalence at follow-up. 
Table 1. Participant demographic characteristics.

\begin{tabular}{|c|c|}
\hline Characteristic & Value $(\mathrm{N}=8392)$ \\
\hline Age (years), mean (SD) & $47.53(13.83)$ \\
\hline \multicolumn{2}{|l|}{ Gender $(n=7303), n(\%)$} \\
\hline Female & $6129(83.92)$ \\
\hline Male & $1147(15.71)$ \\
\hline Other & $27(0.37)$ \\
\hline \multicolumn{2}{|l|}{ Ethnicity $(n=6774), n(\%)$} \\
\hline Hispanic & $436(6.44)$ \\
\hline Non-Hispanic & $6338(93.56)$ \\
\hline \multicolumn{2}{|l|}{ Race $(n=7338), n(\%)$} \\
\hline White & $6586(91.75)$ \\
\hline Black or African American & $231(3.22)$ \\
\hline Asian & $216(3.01)$ \\
\hline Native American or Alaska Native & $83(1.16)$ \\
\hline Native Hawaiian or Pacific Islander & $27(0.38)$ \\
\hline Other & $195(2.72)$ \\
\hline \multicolumn{2}{|l|}{ Income (US \$) (n=6949), n (\%) } \\
\hline$\leq 20,000$ & $212(3.05)$ \\
\hline $21,000-40,000$ & $402(5.79)$ \\
\hline $41,000-60,000$ & $705(10.15)$ \\
\hline $61,000-80,000$ & $942(13.56)$ \\
\hline $81,000-100,000$ & $1055(15.18)$ \\
\hline$>100,000$ & $3633(52.28)$ \\
\hline \multicolumn{2}{|l|}{ Education level $(n=7319), n(\%)$} \\
\hline $11^{\text {th }}$ grade or less & $8(0.11)$ \\
\hline High school or General Educational Development & $161(2.20)$ \\
\hline Some college & $826(11.29)$ \\
\hline 2-year college or technical degree & $2670(36.48)$ \\
\hline Bachelor's degree & $424(5.79)$ \\
\hline Graduate degree & $3230(44.13)$ \\
\hline \multicolumn{2}{|l|}{ Employment (n=7297), n (\%) } \\
\hline Employed & $5084(69.67)$ \\
\hline Retired & $1012(13.87)$ \\
\hline Unemployed & $477(6.54)$ \\
\hline Homemaker & $306(4.19)$ \\
\hline Unable to work & $252(3.45)$ \\
\hline Student & $166(2.27)$ \\
\hline \multicolumn{2}{|l|}{ Rural-urban commuting area $(\mathrm{RUCA})$ code $(n=7044), n(\%)$} \\
\hline 1: Metropolitan area core: primary flow within an Urbanized Area (UA) & $6038(85.73)$ \\
\hline 2: Metropolitan area high commuting: primary flow $30 \%$ or more to a UA & $409(5.83)$ \\
\hline 3: Metropolitan area low commuting: primary flow $10 \%$ to $30 \%$ to a UA & $23(0.29)$ \\
\hline 4: Micropolitan area core: primary flow within an Urban Cluster (UC) of 10,000 to 49,999 (large UC) & $295(4.18)$ \\
\hline 5: Micropolitan high commuting: primary flow $30 \%$ or more to a large UC & $42(0.63)$ \\
\hline
\end{tabular}




\begin{tabular}{lc}
\hline Characteristic & Value $(\mathrm{N}=8392)$ \\
\hline 6: Micropolitan low commuting: primary flow $10 \%$ to $30 \%$ to a large UC & $9(0.11)$ \\
7: Small town core: primary flow within a UC of 2500 to 9999 (small UC) & $121(1.69)$ \\
8: Small town high commuting: primary flow 30\% or more to a small UC & $11(0.24)$ \\
9: Small town low commuting: primary flow 10\% to 30\% to a small UC & $11(0.22)$ \\
10: Rural areas: primary flow to a tract outside a UA or UC & $85(1.19)$ \\
\hline
\end{tabular}

\section{Statistical Analysis}

Descriptive statistics and frequencies were computed to describe sample characteristics. Unadjusted and adjusted regression models were used to examine the association between rural or urban status (ie, RUCA code) and (1) psychological outcomes (ie, stress, depression, and anxiety), (2) pre-COVID-19 and current physical activity, and (3) perceived effects of COVID-19 on stress, mental health, and physical activity, controlling for demographics (ie, gender, age, ethnicity, race, income, education, employment, self-reported Calm app use, and statewide COVID-19 prevalence at baseline). To assess changes in outcomes over time, repeated-measures analyses of variance (ANOVAs) were used. Models included baseline and follow-up outcomes (ie, stress, depression, anxiety, effect of COVID-19 on stress, effect of COVID-19 on mental health, and effect of COVID-19 on physical activity) as within-subjects factors, rural and urban statuses as between-subjects factors, and gender, age, ethnicity, race, income, education, employment, self-reported Calm app use, COVID-19 state baseline (ie, high statewide COVID-19 prevalence at baseline), and COVID-19 state change (ie, change from low statewide COVID-19 prevalence at baseline to high statewide COVID-19 prevalence at follow-up) as covariates. To further assess the effect of rural or urban status and changes in statewide COVID-19 prevalence on changes in outcomes over time, we tested a three-way interaction with time, COVID-19 statewide prevalence, and rural or urban status. All statistical analyses were performed using SPSS, version 26.0 (IBM Corp), with significance inferred at $P<.05$.

\section{Results}

There were total of 8392 participants. Most participants were female (7041/8392, 83.9\%), non-Hispanic (7855/8392, 93.6\%), and White $(7704 / 8392,91.8 \%)$; had high socioeconomic status (income $\geq$ US \$100,000: 4389/8392, 52.3\%; bachelor's degree or higher: $7251 / 8392,86.4 \%$ ); and resided in a metropolitan area core (RUCA 1: 7192/8392, 85.7\%) (Table 1).

As shown in Table 2, at baseline, participants reported moderate stress (mean score 18.12, SD 6.29), where a score of 14 to 26 is considered moderate [23,24]; borderline abnormal levels of depression (mean score $8.89, \mathrm{SD} 4.12$ ), where a score of 8 to 10 is considered borderline abnormal [20]; normal levels of anxiety (mean score 5.87, SD 3.61), where a score of 0 to 7 is considered normal [20]; and being physically active (mean 4.89 days/week, SD 2.29).

Table 2. Baseline psychological outcomes, physical activity, and perceived effects of COVID-19.

\begin{tabular}{|c|c|}
\hline Outcome, physical activity, or effect & Mean (SD) \\
\hline \multicolumn{2}{|l|}{ Psychological outcome } \\
\hline Stress score ${ }^{a}$ & $18.12(6.29)$ \\
\hline Depression score ${ }^{\mathrm{b}}$ & $8.89(4.12)$ \\
\hline Anxiety score ${ }^{\mathrm{b}}$ & $5.87(3.61)$ \\
\hline \multicolumn{2}{|l|}{ Physical activity (days/week) ${ }^{c}$} \\
\hline Pre-COVID-19 physical activity & $4.87(2.04)$ \\
\hline Current physical activity & $4.89(2.29)$ \\
\hline \multicolumn{2}{|l|}{ Perceived effect $^{\mathrm{d}}$ of COVID-19 on: } \\
\hline Stress & $1.82(0.80)$ \\
\hline Mental health & $2.07(0.83)$ \\
\hline Physical activity & $2.72(1.29)$ \\
\hline
\end{tabular}

${ }^{a}$ Stress was measured using the Perceived Stress Scale; summed scores range from 0 to 40, with higher scores indicating higher levels of perceived stress.

${ }^{\mathrm{b}}$ Depression and anxiety were measured using the depression and anxiety subscales, respectively, of the Hospital Anxiety and Depression Scale; summed scores range from 0 to 21 (normal: 0-7; borderline abnormal: 8-10; abnormal: 11-21).

${ }^{c}$ Participants self-reported how many days per week (on a scale of 0 to 7 ) of physical activity they participated in.

${ }^{\mathrm{d}}$ Participants responded to investigator-developed items; response scores range from 1 to 5 , with higher scores indicating higher perceived effects of COVID-19. 
As shown in Table 3, in both unadjusted and adjusted regression models, rural or urban status was not significantly associated with baseline stress, depression, anxiety, physical activity, or perceived effects of COVID-19 on stress, mental health, and physical activity. In our alternative analyses where we treated
RUCA as a continuous variable, we also found no significant associations between rural or urban status and mental health outcomes, physical activity, or perceived effects of COVID-19 (Multimedia Appendix 1).

Table 3. Unadjusted and adjusted regression models exploring associations between rural or urban status and psychological outcomes, physical activity, and perceived effects of COVID-19.

\begin{tabular}{|c|c|c|}
\hline Model & Rural or urban difference, $\beta$ (SE) & $P$ value \\
\hline \multicolumn{3}{|l|}{ Unadjusted model } \\
\hline \multicolumn{3}{|l|}{ Psychological outcome } \\
\hline Stress & $0.30(0.22)$ & .09 \\
\hline Depression & $0.15(0.14)$ & .29 \\
\hline Anxiety & $-0.15(0.12)$ & .24 \\
\hline \multicolumn{3}{|l|}{ Physical activity } \\
\hline Pre-COVID-19 physical activity & $0.08(0.07)$ & .27 \\
\hline Current physical activity & $0.08(0.08)$ & .31 \\
\hline \multicolumn{3}{|l|}{ Perceived effect of COVID-19 on: } \\
\hline Stress & $-0.05(0.03)$ & .10 \\
\hline Mental health & $-0.07(0.03)$ & .11 \\
\hline Physical activity & $-0.05(0.04)$ & .29 \\
\hline \multicolumn{3}{|l|}{ Adjusted model $^{\mathbf{a}}$} \\
\hline \multicolumn{3}{|l|}{ Psychological outcome } \\
\hline Stress & $0.02(0.22)$ & .91 \\
\hline Depression & $0.07(0.15)$ & .64 \\
\hline Anxiety & $-0.24(0.13)$ & .07 \\
\hline \multicolumn{3}{|l|}{ Physical activity } \\
\hline Pre-COVID-19 physical activity & $0.12(0.08)$ & .10 \\
\hline Current physical activity & $0.16(0.09)$ & .05 \\
\hline \multicolumn{3}{|l|}{ Perceived effect of COVID-19 on: } \\
\hline Stress & $-0.05(0.03)$ & .09 \\
\hline Mental health & $-0.07(0.03)$ & .22 \\
\hline Physical activity & $-0.02(0.05)$ & .67 \\
\hline
\end{tabular}

\footnotetext{
${ }^{\mathrm{a}}$ The model controlled for gender, age, ethnicity, race, income, education, employment, Calm app use, and statewide COVID-19 prevalence at baseline.

As shown in Table 4, results from repeated-measures ANOVAs showed no significant changes in stress or perceived effects of COVID-19 on stress and mental health from baseline to follow-up. In models of depression, anxiety, and effect of COVID-19 on physical activity, there were significant main effects of time, which showed that, overall, symptoms of depression, anxiety, and perceived effect of COVID-19 on physical activity decreased from baseline to follow-up. Furthermore, there were significant main effects of age on most outcomes, which showed that, at baseline, older participants had greater stress, depression, and anxiety; engaged in more physical activity; and perceived that COVID-19 had a greater effect on their stress and mental health. There were also significant time $\times$ age interactions in models of stress and anxiety, such that older participants had smaller decreases in

stress and anxiety over time than did younger participants. Results of full models with covariates can be found in Multimedia Appendix 1.

There were no significant main effects of rural or urban status, statewide COVID-19 prevalence at baseline, or change in statewide COVID-19 prevalence in any of the models (Table 4). In addition, there were no significant time $\times$ rural or urban status interactions, time $\times$ statewide COVID-19 prevalence at baseline interactions, or time $\times$ change in statewide COVID-19 prevalence interactions (Table 4). Lastly, we found no significant three-way interactions (ie, time $\times$ statewide COVID-19 prevalence at baseline $\times$ rural or urban status and time $\times$ change in statewide COVID-19 prevalence $\times$ rural or urban status).
} 
Table 4. Results of repeated-measures analyses of variance for baseline and follow-up study outcomes ${ }^{\mathrm{a}}$.

\begin{tabular}{|c|c|c|c|c|}
\hline Model & $\begin{array}{l}\text { Baseline score, } \\
\text { mean (SD) }\end{array}$ & $\begin{array}{l}\text { Follow-up score, } \\
\text { mean (SD) }\end{array}$ & $F$ test $(d f)$ & $P$ value \\
\hline Stress & $18.013(6.174)$ & $17.180(6.322)$ & & \\
\hline Time & & & $0.049(1,2636)$ & .83 \\
\hline Rural-urban commuting area (RUCA) & & & $0.160(1,2636)$ & .69 \\
\hline Time $\times$ RUCA & & & $0.000(1,2636)$ & .99 \\
\hline COVID-19 state baseline & & & $1.078(1,2636)$ & .30 \\
\hline Time $\times$ COVID-19 state baseline & & & $0.015(1,2636)$ & .90 \\
\hline Time $\times$ COVID-19 state baseline $\times$ RUCA & & & $0.116(1,2636)$ & .73 \\
\hline COVID-19 state change & & & $0.069(1,2636)$ & .79 \\
\hline Time $\times$ COVID- 19 state change & & & $0.006(1,2636)$ & .94 \\
\hline Time $\times$ COVID- 19 state change $\times$ RUCA & & & $0.168(1,2636)$ & .68 \\
\hline Depression & $8.832(4.097)$ & $8.396(4.156)$ & & \\
\hline Time & & & $3.868(1,2613)$ & .049 \\
\hline RUCA & & & $0.017(1,2613)$ & .90 \\
\hline Time $\times$ RUCA & & & $0.339(1,2613)$ & .56 \\
\hline COVID-19 state baseline & & & $0.386(1,2613)$ & .54 \\
\hline Time $\times$ COVID-19 state baseline & & & $0.112(1,2613)$ & .74 \\
\hline Time $\times$ COVID- 19 state baseline $\times$ RUCA & & & $0.047(1,2613)$ & .83 \\
\hline COVID-19 state change & & & $0.038(1,2613)$ & .85 \\
\hline Time $\times$ COVID- 19 state change & & & $0.519(1,2613)$ & .47 \\
\hline Time $\times$ COVID- 19 state change $\times$ RUCA & & & $0.183(1,2613)$ & .67 \\
\hline Anxiety & $5.755(3.590)$ & $5.474(3.663)$ & & \\
\hline Time & & & $4.648(1,2613)$ & .03 \\
\hline RUCA & & & $0.409(1,2613)$ & .52 \\
\hline Time $\times$ RUCA & & & $0.353(1,2613)$ & .55 \\
\hline COVID-19 state baseline & & & $0.022(1,2613)$ & .88 \\
\hline Time $\times$ COVID-19 state baseline & & & $0.626(1,2613)$ & .43 \\
\hline Time $\times$ COVID- 19 state baseline $\times$ RUCA & & & $1.121(1,2613)$ & .29 \\
\hline COVID-19 state change & & & $0.211(1,2613)$ & .65 \\
\hline Time $\times$ COVID-19 state change & & & $0.010(1,2613)$ & .92 \\
\hline Time $\times$ COVID- 19 state change $\times$ RUCA & & & $0.310(1,2613)$ & .58 \\
\hline Physical activity & $4.930(2.305)$ & $4.950(2.243)$ & & \\
\hline Time & & & $0.188(1,2519)$ & .67 \\
\hline RUCA & & & $1.227(1,2519)$ & .27 \\
\hline Time $\times$ RUCA & & & $1.248(1,2519)$ & .26 \\
\hline COVID-19 state baseline & & & $0.046(1,2519)$ & .83 \\
\hline Time $\times$ COVID -19 state baseline & & & $0.659(1,2519)$ & .42 \\
\hline Time $\times$ COVID- 19 state baseline $\times$ RUCA & & & $0.779(1,2519)$ & .38 \\
\hline COVID-19 state change & & & $0.000(1,2519)$ & $>.99$ \\
\hline Time $\times$ COVID- 19 state change & & & $2.061(1,2519)$ & .15 \\
\hline Time $\times$ COVID- 19 state change $\times$ RUCA & & & $3.550(1,2519)$ & .06 \\
\hline Effect of COVID-19 on stress & $1.830(0.821)$ & $1.790(0.783)$ & & \\
\hline
\end{tabular}




\begin{tabular}{|c|c|c|c|c|}
\hline Model & $\begin{array}{l}\text { Baseline score, } \\
\text { mean (SD) }\end{array}$ & $\begin{array}{l}\text { Follow-up score, } \\
\text { mean (SD) }\end{array}$ & $F$ test $(d f)$ & $P$ value \\
\hline Time & & & $0.717(1,2487)$ & .40 \\
\hline RUCA & & & $0.189(1,2487)$ & .66 \\
\hline Time $\times$ RUCA & & & $3.344(1,2487)$ & .07 \\
\hline COVID-19 state baseline & & & $0.935(1,2487)$ & .33 \\
\hline Time $\times$ COVID-19 state baseline & & & $2.506(1,2487)$ & .11 \\
\hline Time $\times$ COVID-19 state baseline $\times$ RUCA & & & $1.077(1,2487)$ & .30 \\
\hline COVID-19 state change & & & $0.002(1,2487)$ & .97 \\
\hline Time $\times$ COVID- 19 state change & & & $1.203(1,2487)$ & .27 \\
\hline Time $\times$ COVID- 19 state change $\times$ RUCA & & & $0.084(1,2487)$ & .77 \\
\hline Effect of COVID-19 on mental health & $2.110(0.846)$ & $2.030(0.821)$ & & \\
\hline Time & & & $0.081(1,2481)$ & .78 \\
\hline RUCA & & & $0.138(1,2481)$ & .71 \\
\hline Time $\times$ RUCA & & & $0.002(1,2481)$ & .96 \\
\hline COVID-19 state baseline & & & $0.123(1,2481)$ & .73 \\
\hline Time $\times$ COVID-19 state baseline & & & $0.255(1,2481)$ & .61 \\
\hline Time $\times$ COVID-19 state baseline $\times$ RUCA & & & $0.510(1,2481)$ & .48 \\
\hline COVID-19 state change & & & $0.069(1,2481)$ & .79 \\
\hline Time $\times$ COVID- 19 state change & & & $0.006(1,2481)$ & .94 \\
\hline Time $\times$ COVID- 19 state change $\times$ RUCA & & & $0.168(1,2481)$ & .68 \\
\hline Effect of COVID-19 on physical activity & $2.740(1.278)$ & $2.670(1.261)$ & & \\
\hline Time & & & $3.868(1,2483)$ & .049 \\
\hline RUCA & & & $0.017(1,2483)$ & .90 \\
\hline Time $\times$ RUCA & & & $0.339(1,2483)$ & .56 \\
\hline COVID-19 state baseline & & & $0.386(1,2483)$ & .54 \\
\hline Time $\times$ COVID-19 state baseline & & & $0.112(1,2483)$ & .74 \\
\hline Time $\times$ COVID-19 state baseline $\times$ RUCA & & & $0.047(1,2483)$ & .83 \\
\hline COVID-19 state change & & & $0.038(1,2483)$ & .85 \\
\hline Time $\times$ COVID- 19 state change & & & $0.519(1,2483)$ & .47 \\
\hline Time $\times$ COVID- 19 state change $\times$ RUCA & & & $0.183(1,2483)$ & .67 \\
\hline
\end{tabular}

${ }^{\mathrm{a}}$ Baseline and follow-up mean (SD) values were only reported for each within-subjects factor, while $F$ test $(d f)$ values and $P$ values were only reported for each between-subjects factor and interaction effects.

\section{Discussion}

\section{Principal Findings}

In this study, we explored associations between rural or urban status, psychological outcomes, and physical activity among meditation app users. We additionally explored associations between rural or urban status and perceived effects of the ongoing COVID-19 pandemic on stress, mental health, and physical activity, as well as changes in study outcomes among rural versus urban app users over time. Overall, we found no significant associations between rural or urban status, psychological outcomes, physical activity, or perceived effects of COVID-19 on stress, mental health, and physical activity at baseline. We also found that there were significant decreases in depression, anxiety, and perceived effects of COVID-19 on physical activity over time, but there were no significant changes in stress, physical activity participation, or perceived effects of COVID-19 on stress or mental health. Additionally, there were no significant time $\times$ statewide COVID-19 prevalence $\times$ rural or urban status interactions.

Our findings generally contrast previous studies that demonstrated rural and urban differences in physical and mental health outcomes and behaviors [1-6]. Specifically, some studies found a higher prevalence of major mental disorders, including mood and anxiety disorders, in urban areas [2,25]. For example, one literature review concluded that living in urban cities was associated with a considerably higher risk for schizophrenia [2]; in addition, another meta - analysis of urban and rural 
differences in psychiatric disorders, which was conducted on data taken from 20 population survey studies, found that prevalence rates for psychiatric disorders were significantly higher in urban areas compared with rural areas [25]. Other studies found a higher prevalence of depression in rural areas [16] and have shown that rural residents receive less mental health treatment despite poorer mental health [17]. For example, a cross-sectional study using the National Health Interview Survey found that depression prevalence was significantly higher among rural populations than among urban populations [16]. Another study using data from the Medical Expenditure Panel Survey concluded that the odds of receiving any mental health treatment and specialized mental health treatment were $47 \%$ and $72 \%$ higher, respectively, for metropolitan residents compared to those living in the most rural settings [17].

Regarding health behaviors such as physical activity, rural residents have consistently been less physically active compared to their urban counterparts, which has been attributed to increased barriers and limited physical activity resources in rural areas $[6,18,26]$. For example, an analysis of data from the Behavioral Risk Factor Surveillance System, conducted among 398,208 adults, demonstrated that residents of nonmetropolitan counties had a lower prevalence of meeting national physical activity guidelines compared to their metropolitan counterparts [18]. These referenced studies exploring rural and urban differences in health outcomes and behaviors have generally been conducted among adults outside the context of participation in a behavioral health intervention or use of an available behavioral health program, unlike this study, which explored these differences among users of a meditation app.

This was the first study, to our knowledge, to assess rural and urban differences among meditation app users during the COVID-19 pandemic. Our results suggest that the rural residents in our sample who use meditation apps have access to tools that may address rural health disparities. For example, meditation app users need to own a mobile device and have internet access, which are social determinants of health [27]. Furthermore, mobile meditation app users likely possess digital literacy (ie, the ability to understand and utilize electronic resources or identify, access, and use electronic information from networks as well as having the skills to decipher texts, sounds, or images) $[28,29]$. Lastly, seeking out, downloading, and using a meditation app to manage health-related outcomes and symptoms reflect health literacy (ie, the capacity to obtain, process, and understand basic health information and services needed to make appropriate health decisions) [30], which is associated with mHealth app use [31]. Overall, a combination of mobile device ownership, internet access, and digital and health literacy may be important tools for addressing rural health disparities. The use of a meditation app, specifically, may further contribute to addressing disparities via improvement of mental health outcomes and health behaviors among rural residents. Thus, research should continue to explore and establish the use of meditation apps for health promotion in both rural and urban populations.

There were no associations between rural or urban status and perceived effects of the COVID-19 pandemic on stress, mental health, or physical activity. Emerging literature has demonstrated that stress and symptoms of anxiety and depression are common psychological responses to COVID-19 [32], but studies have not yet focused on rural populations or assessed rural and urban differences in perceived effects of COVID-19. Our results suggest that during this ongoing pandemic, mobile device ownership, internet access, digital and health literacy, and meditation app use may address disparities related to the perceived effects of COVID-19 on health and health behaviors. This further reinforces the importance of using meditation apps among both rural and urban populations. However, it is important to note that our sample largely consisted of non-Hispanic White women with relatively high socioeconomic status, and may not be reflective of rural or urban mHealth app users from lower-income or racial and ethnic minority backgrounds, and the benefits of meditation apps and their potential for reducing disparities must be confirmed with studies in these underrepresented populations. Furthermore, given that the majority of our sample was urban, the absence of rural and urban differences in outcomes must be confirmed in a larger, more representative sample.

We found overall decreases in depression, anxiety, and perceived effect of COVID-19 on physical activity and no overall changes from baseline to follow-up in stress, physical activity, or perceived effects of COVID-19 on stress and mental health. Prior studies have generally demonstrated a reduction in physical activity since the start of the pandemic [33-35], and our results suggest that meditation apps may be a potential strategy for counteracting decreases in physical activity during the pandemic. Studies assessing changes in psychological outcomes over the course of the COVID-19 pandemic have resulted in mixed findings; for example, one study found increases in depression and decreases in anxiety in Argentina [36], another study found increases in anxiety in the United Kingdom [37], and another found no changes in stress, depression, or anxiety in China [38]. However, there have been limited studies longitudinally assessing changes in mental health outcomes during the pandemic in the United States. Our results provide preliminary evidence that despite the increase in state-level COVID-19 cases in the United States, there may not be negative cumulative effects of the pandemic on psychological outcomes and health behaviors among our sample (ie, mainly urban, White, female, and of high socioeconomic status) of meditation app users. However, further studies are necessary to understand longer-term effects of COVID-19 in this group, and to establish whether meditation delivered via mobile apps is an effective strategy for promoting health outcomes and behaviors over the course of the pandemic. Our results further suggest no significant effect of rural or urban status and statewide COVID-19 prevalence-both at baseline and change from baseline to follow-up - on changes in outcomes over time. However, more research is needed to elucidate time-varying changes in psychological and behavioral outcomes between rural and urban meditation app users, which could help to identify at-risk groups for interventions.

\section{Limitations}

This study was the first to explore associations between rural or urban status and psychological outcomes, physical activity, and perceived effects of COVID-19 on stress, mental health, 
and physical activity among meditation app users. However, there were limitations that should be noted. Our sample was primarily female, non-Hispanic, and White and had high socioeconomic status, limiting generalizability of findings to other populations, such as racial and ethnic minorities and low-income individuals. The majority of our sample was further categorized as urban, with $85.7 \%$ of participants residing in the US Department of Agriculture's definition of a metropolitan core area (ie, RUCA 1). Although these sample demographics are frequently reflected in research assessing rural and urban differences, with studies including $80.0 \%$ to $88.7 \%$ of participants residing in RUCA 1 or the highest level of urbanization [3,39-41], future research in this area should aim to include a larger proportion of rural residents.

Furthermore, all outcomes in this study were assessed via self-report measures, which are subject to social desirability and recall bias. In addition, another limitation was the use of investigator-developed survey questions as opposed to validated questionnaires. However, for some outcomes, such as the extent to which COVID-19 impacted outcomes, there were no validated instruments when this study was conducted. With regard to behavioral outcomes (ie, physical activity and meditation), future studies in this area should aim to use device-based measures. Physical activity, in particular, is one of the outcomes to be particularly cautious about when interpreting study results. Physical activity is often overreported by participants in research studies, with respondents reporting higher rates of, or more frequent, activity than actual behavior warrants, which in turn causes self-reported measures of physical activity to suffer from low validity [42]. Thus, given our self-reported items for measuring physical activity in this study, it is important to note that these results are preliminary and must be validated in larger studies using device-based measures, which may be complemented with self-report measures to provide activity type and context. Another limitation was inherent to the study design, which included a national survey conducted among a nonrandom convenience sample. Although this study was able to contribute novel, preliminary findings, future research should assess associations between rural or urban status and health outcomes among mHealth app users in a randomized controlled trial in order to decrease bias and increase scientific rigor. Lastly, although we included total state-level COVID-19 cases over time in the analyses, a combination of additional factors, such as lockdowns, hospitalizations, and deaths, may have had an influence on participants' psychological and physical activity outcomes and perceived effects of COVID-19 on health and behaviors. Further research is also needed to establish whether cumulative effects of the pandemic will become more apparent after a longer period of time.

\section{Conclusions}

Overall, we did not find any associations between rural or urban status and psychological outcomes (ie, stress, depression, and anxiety), pre-COVID-19 and current physical activity, or perceived effects of COVID-19 on stress, mental health, and physical activity among users of a meditation app. Rural or urban status does not appear to drive differences in outcomes among meditation app users, and the use of mHealth apps should continue to be explored as a health promotion strategy in both rural and urban populations. Our results also did not show negative cumulative effects of COVID-19 on psychological outcomes and physical activity in our sample, and research should continue to explore meditation apps as a health promotion strategy during the pandemic.

\section{Acknowledgments}

The researchers would like to thank all the participants who took part in this study.

\section{Authors' Contributions}

JH and CS conceptualized the study and guided the study design. NB conducted the data analyses and drafted the manuscript. MP assisted with data analyses and interpretation of results. All authors critically reviewed and edited the manuscript and approved its submission.

\section{Conflicts of Interest}

JH is currently consulting as the Director of Science at Calm. JH has been conducting research with Calm as a partner for 6 years prior to the Director of Science role. JH directs the Scientific Advisory Board at Calm. JH has no stock in Calm and receives no financial incentives from the sales of Calm.

\section{Multimedia Appendix 1}

Unadjusted and adjusted regression models exploring associations between rural-urban status (ie, continuous rural-urban commuting area [RUCA]) and mental health outcomes, physical activity, and perceived effects of COVID-19 as well as results of repeated measures analyses.

[XLSX File (Microsoft Excel File), 30 KB-Multimedia Appendix 1]

\section{References}

1. Henley SJ, Anderson RN, Thomas CC, Massetti GM, Peaker B, Richardson LC. Invasive cancer incidence, 2004-2013, and deaths, 2006-2015, in nonmetropolitan and metropolitan counties - United States. MMWR Surveill Summ 2017 Jul 07;66(14):1-13 [FREE Full text] [doi: 10.15585/mmwr.ss6614a1] [Medline: 28683054] 
2. Gruebner O, Rapp M, Adli M, Kluge U, Galea S, Heinz A. Cities and mental health. Dtsch Arztebl Int 2017 Feb 24;114(8):121-127 [FREE Full text] [doi: 10.3238/arztebl.2017.0121] [Medline: 28302261]

3. Kozhimannil KB, Interrante JD, Henning-Smith C, Admon LK. Rural-urban differences in severe maternal morbidity and mortality in the US, 2007-15. Health Aff (Millwood) 2019 Dec;38(12):2077-2085. [doi: 10.1377/hlthaff.2019.00805] [Medline: 31794322$]$

4. O'Connor A, Wellenius G. Rural-urban disparities in the prevalence of diabetes and coronary heart disease. Public Health 2012 Oct;126(10):813-820. [doi: 10.1016/j.puhe.2012.05.029] [Medline: 22922043]

5. Suliga E. Nutritional behaviours of pregnant women in rural and urban environments. Ann Agric Environ Med 2015;22(3):513-517 [FREE Full text] [doi: 10.5604/12321966.1167725] [Medline: 26403126]

6. Whitfield GP, Carlson SA, Ussery EN, Fulton JE, Galuska DA, Petersen R. Trends in meeting physical activity guidelines among urban and rural dwelling adults - United States, 2008-2017. MMWR Morb Mortal Wkly Rep 2019 Jun 14;68(23):513-518 [FRE Full text] [doi: 10.15585/mmwr.mm6823a1] [Medline: 31194722]

7. Chandrashekar P. Do mental health mobile apps work: Evidence and recommendations for designing high-efficacy mental health mobile apps. Mhealth 2018;4:6 [FREE Full text] [doi: 10.21037/mhealth.2018.03.02] [Medline: 29682510]

8. Han M, Lee E. Effectiveness of mobile health application use to improve health behavior changes: A systematic review of randomized controlled trials. Healthc Inform Res 2018 Jul;24(3):207-226 [FREE Full text] [doi: 10.4258/hir.2018.24.3.207] [Medline: 30109154]

9. Iribarren SJ, Cato K, Falzon L, Stone PW. What is the economic evidence for mHealth? A systematic review of economic evaluations of mHealth solutions. PLoS One 2017;12(2):e0170581 [FREE Full text] [doi: 10.1371/journal.pone.0170581] [Medline: 28152012]

10. Healthcare facilities: Managing operations during the COVID-19 pandemic. Centers for Disease Control and Prevention. 2020. URL: https://www.cdc.gov/coronavirus/2019-ncov/hcp/guidance-hcf.html [accessed 2020-11-05]

11. Mallow JA, Theeke LA, Barnes ER, Whetsel T, Mallow BK. Using mHealth tools to improve rural diabetes care guided by the chronic care model. Online J Rural Nurs Health Care 2014;14(1):43-65 [FREE Full text] [doi: 10.14574/ojrnhc.v14i1.276] [Medline: 26029005]

12. Yin Z, Lesser J, Paiva KA, Zapata J, Moreno-Vasquez A, Grigsby TJ, et al. Using mobile health tools to engage rural underserved individuals in a diabetes education program in South Texas: Feasibility study. JMIR Mhealth Uhealth 2020 Mar 24;8(3):e16683 [FREE Full text] [doi: 10.2196/16683] [Medline: 32207694]

13. Ostafin BD, Chawla N, Bowen S, Dillworth TM, Witkiewitz K, Marlatt GA. Intensive mindfulness training and the reduction of psychological distress: A preliminary study. Cogn Behav Pract 2006 Aug;13(3):191-197. [doi: 10.1016/j.cbpra.2005.12.001]

14. Edenfield TM, Saeed SA. An update on mindfulness meditation as a self-help treatment for anxiety and depression. Psychol Res Behav Manag 2012 Oct:131. [doi: 10.2147/prbm.s34937]

15. Bigliassi M, Bertuzzi R. Exploring the use of meditation as a valuable tool to counteract sedentariness. Front Psychol 2020;11:299 [FREE Full text] [doi: 10.3389/fpsyg.2020.00299] [Medline: 32158418]

16. Probst JC, Laditka SB, Moore CG, Harun N, Powell MP, Baxley EG. Rural-urban differences in depression prevalence: Implications for family medicine. Fam Med 2006 Oct;38(9):653-660 [FREE Full text] [Medline: 17009190]

17. Hauenstein EJ, Petterson S, Rovnyak V, Merwin E, Heise B, Wagner D. Rurality and mental health treatment. Adm Policy Ment Health 2007 May;34(3):255-267. [doi: 10.1007/s10488-006-0105-8] [Medline: 17165139]

18. Matthews KA, Croft JB, Liu Y, Lu H, Kanny D, Wheaton AG, et al. Health-related behaviors by urban-rural county classification - United States, 2013. MMWR Surveill Summ 2017 Feb 03;66(5):1-8 [FREE Full text] [doi: 10.15585/mmwr.ss6605a1] [Medline: 28151923]

19. Lee E. Review of the psychometric evidence of the perceived stress scale. Asian Nurs Res (Korean Soc Nurs Sci) 2012 Dec;6(4):121-127 [FREE Full text] [doi: 10.1016/j.anr.2012.08.004] [Medline: 25031113]

20. Zigmond AS, Snaith RP. The hospital anxiety and depression scale. Acta Psychiatr Scand 1983 Jun;67(6):361-370. [doi: $\underline{10.1111 / j .1600-0447.1983 . t b 09716 . x}$ ] [Medline: $\underline{6880820}$ ]

21. Rural-urban commuting area codes. United States Department of Agriculture, Economic Research Service. 2020. URL: https://www.ers.usda.gov/data-products/rural-urban-commuting-area-codes/ [accessed 2020-11-06]

22. United States COVID-19 cases and deaths by state over time. Centers for Disease Control and Prevention. 2020. URL: https://data.cdc.gov/Case-Surveillance/United-States-COVID-19-Cases-and-Deaths-by-State-o/9mfq-cb36 [accessed 2020-11-05]

23. Menon P, Chaudhury S, Saldanha D, Sahu S, Singh V, Pathak V. Stress levels and its association with self-harm and risk-taking behavior in medical undergraduates. Ind Psychiatry J 2018;27(1):41-46 [FREE Full text] [doi: 10.4103/ipj.ipj 31 18] [Medline: $\underline{30416290]}$

24. Sahu M, Gandhi S, Sharma MK, Marimuthu P. Perceived stress and resilience and their relationship with the use of mobile phone among nursing students. Invest Educ Enferm 2019 Sep;37(3):e05 [FREE Full text] [doi: 10.17533/udea.iee.v37n3e05] [Medline: $\underline{\text { 31830403] }}$

25. Peen J, Schoevers RA, Beekman AT, Dekker J. The current status of urban-rural differences in psychiatric disorders. Acta Psychiatr Scand 2010 Feb;121(2):84-93. [doi: 10.1111/j.1600-0447.2009.01438.x] [Medline: 19624573] 
26. Gilbert AS, Duncan DD, Beck AM, Eyler AA, Brownson RC. A qualitative study identifying barriers and facilitators of physical activity in rural communities. J Environ Public Health 2019;2019:7298692 [FREE Full text] [doi: 10.1155/2019/7298692] [Medline: $\underline{31341485}$ ]

27. Daniel H, Bornstein SS, Kane GC. Addressing social determinants to improve patient care and promote health equity: An American College of Physicians position paper. Ann Intern Med 2018 Apr 17;168(8):577. [doi: 10.7326/m17-2441]

28. Bawden D. Information and digital literacies: A review of concepts. J Doc 2001 Apr;57(2):218-259. [doi: 10.1108/eum0000000007083]

29. Noh Y. A study on the effect of digital literacy on information use behavior. J Libr Inf Sci 2016 Jul 09;49(1):26-56. [doi: $10.1177 / 0961000615624527]$

30. Berkman ND, Davis TC, McCormack L. Health literacy: What is it? J Health Commun 2010;15 Suppl 2:9-19. [doi: 10.1080/10810730.2010.499985] [Medline: 20845189]

31. Mackert M, Mabry-Flynn A, Champlin S, Donovan EE, Pounders K. Health literacy and health information technology adoption: The potential for a new digital divide. J Med Internet Res 2016 Oct 04;18(10):e264 [FREE Full text] [doi: 10.2196/jmir.6349] [Medline: 27702738]

32. Rajkumar RP. COVID-19 and mental health: A review of the existing literature. Asian J Psychiatr 2020 Aug;52:102066 [FREE Full text] [doi: 10.1016/j.ajp.2020.102066] [Medline: 32302935]

33. Duncan GE, Avery AR, Seto E, Tsang S. Perceived change in physical activity levels and mental health during COVID-19: Findings among adult twin pairs. PLoS One 2020;15(8):e0237695 [FREE Full text] [doi: 10.1371/journal.pone.0237695] [Medline: 32790745$]$

34. Tison GH, Avram R, Kuhar P, Abreau S, Marcus GM, Pletcher MJ, et al. Worldwide effect of COVID-19 on physical activity: A descriptive study. Ann Intern Med 2020 Nov 03;173(9):767-770. [doi: 10.7326/m20-2665]

35. Ingram J, Maciejewski G, Hand CJ. Changes in diet, sleep, and physical activity are associated with differences in negative mood during COVID-19 lockdown. Front Psychol 2020;11:588604 [FREE Full text] [doi: 10.3389/fpsyg.2020.588604] [Medline: 32982903]

36. Canet-Juric L, Andrés ML, Del Valle M, López-Morales H, Poó F, Galli JI, et al. A longitudinal study on the emotional impact cause by the COVID-19 pandemic quarantine on general population. Front Psychol 2020;11:565688 [FREE Full text] [doi: 10.3389/fpsyg.2020.565688] [Medline: $\underline{33071893}$ ]

37. Kwong A, Pearson R, Adams M, Northstone K, Tilling K, Smith D, et al. Mental health before and during the COVID-19 pandemic in two longitudinal UK population cohorts. Br J Psychiatry 2020 Nov 24:1-10 [FREE Full text] [doi:

10.1192/bjp.2020.242] [Medline: 33228822]

38. Wang C, Pan R, Wan X, Tan Y, Xu L, McIntyre RS, et al. A longitudinal study on the mental health of general population during the COVID-19 epidemic in China. Brain Behav Immun 2020 Jul;87:40-48 [FREE Full text] [doi: 10.1016/j.bbi.2020.04.028] [Medline: 32298802]

39. Euler R, Jimenez EY, Sanders S, Kuhlemeier A, Van Horn ML, Cohen D, et al. Rural-urban differences in baseline dietary intake and physical activity levels of adolescents. Prev Chronic Dis 2019 Jan 03;16:E01 [FREE Full text] [doi: 10.5888/pcd16.180200] [Medline: $\underline{30605423}$ ]

40. Segel JE, Lengerich EJ. Rural-urban differences in the association between individual, facility, and clinical characteristics and travel time for cancer treatment. BMC Public Health 2020 Feb 06;20(1):196 [FREE Full text] [doi: 10.1186/s12889-020-8282-z] [Medline: 32028942]

41. Margerison CE, Goldman-Mellor S. Association between rural residence and nonfatal suicidal behavior among California adults: A population-based study. J Rural Health 2019 Mar;35(2):262-269 [FREE Full text] [doi: 10.1111/jrh.12352] [Medline: $\underline{30703850}$ ]

42. Brenner PS, DeLamater JD. Social desirability bias in self-reports of physical activity: Is an exercise identity the culprit? Soc Indic Res 2013 Jun 1;117(2):489-504. [doi: 10.1007/s11205-013-0359-y]

\author{
Abbreviations \\ ANOVA: analysis of variance \\ HADS: Hospital Anxiety and Depression Scale \\ HADS-A: Hospital Anxiety and Depression Scale-anxiety subscale \\ HADS-D: Hospital Anxiety and Depression Scale-depression subscale \\ mHealth: mobile health \\ RUCA: rural-urban commuting area \\ SQL: Structured Query Language
}


Edited by L Buis; submitted 25.11.20; peer-reviewed by J Brewer, AS Aslam, D Szinay, C Emezue; comments to author 08.01.21; revised version received 27.02.21; accepted 20.04.21; published 26.05.21

Please cite as:

Bhuiyan N, Puzia M, Stecher C, Huberty J

Associations Between Rural or Urban Status, Health Outcomes and Behaviors, and COVID-19 Perceptions Among Meditation App Users: Longitudinal Survey Study

JMIR Mhealth Uhealth 2021;9(5):e26037

URL: https://mhealth.jmir.org/2021/5/e26037

doi: $\underline{10.2196 / 26037}$

PMID: $\underline{33900930}$

CNishat Bhuiyan, Megan Puzia, Chad Stecher, Jennifer Huberty. Originally published in JMIR mHealth and uHealth (https://mhealth.jmir.org), 26.05.2021. This is an open-access article distributed under the terms of the Creative Commons Attribution License (https://creativecommons.org/licenses/by/4.0/), which permits unrestricted use, distribution, and reproduction in any medium, provided the original work, first published in JMIR mHealth and uHealth, is properly cited. The complete bibliographic information, a link to the original publication on https://mhealth.jmir.org/, as well as this copyright and license information must be included. 